\title{
Using Finite Forkable DEVS for Decision-Making Based on Time Measured with Uncertainty
}

\author{
Damián Vicino $* \dagger \ddagger$ \\ Université Nice Sophia \\ Antipolis \\ I3S UMR CNRS 7271 \\ INRIA - Sophia Antipolis \\ Carleton University \\ Dept. of Systems and \\ Computer Engineering \\ vicino@i3s.unice.fr
}

\author{
Olivier Dalle ${ }^{*}$ \\ Université Nice Sophia \\ Antipolis \\ I3S UMR CNRS 7271 \\ INRIA - Sophia Antipolis \\ olivier.dalle@unice.fr
}

\author{
Gabriel Wainer ${ }^{\ddagger}$ \\ Carleton University \\ Dept. of Systems and \\ Computer Engineering \\ gwainer@sce.carleton.ca
}

\begin{abstract}
The time-line in Discrete Event Simulation (DES) is a sequence of events defined in a numerable subset of $\mathbb{R}^{+}$. When it comes from an experimental measurement, the timing of these events has a limited precision. This precision is usually well-known and documented for each instruments and procedures used for collecting experimental datas. Therefore, these instruments and procedures produce measurement results expressed using values each associated with an uncertainty quantification, given by uncertainty intervals. Tools have been developed in Continuous Systems modeling for deriving the uncertainty intervals of the final results corresponding to the propagation of the uncertainty intervals being evaluated. These tools cannot be used in DES as they are defined, and no alternative tools that would apply to DES have been developed yet. In this paper, we propose simulation algorithms, based on the Discrete Event System Specification (DEVS) formalism, that can be used to simulate and obtain every possible output and state trajectories of simulations that receive input values with uncertainty quantification. Then, we present a subclass of DEVS models, called Finite Forkable DEVS (FF-DEVS), that can be simulated by the proposed algorithms. This subclass ensures that the simulation is forking only a finite number of processes for each simulation step. Finally, we discuss the

*Université Nice Sophia Antipolis, Laboratoire I3S
UMR CNRS 7271,2000 route des Lucioles, BP 93,
06900 Sophia Antipolis, France
†INRIA - Sophia Antipolis, 2004 Route des Lucioles,
06903 Sophia Antipolis, France
‡Carleton University, Dept. of Systems and Com-
puter Engineering, 1125 Colonel By Dr., Ottawa, ON,
Canada
\end{abstract}

simulation of a traffic light model and show the trajectories obtained when it is subject to input uncertainty.

\section{Categories and Subject Descriptors}

I.6.8 [Types of Simulation]: Discrete event; I.1.2 [Symbolic and algebraic manipulation]: Algorithms; F.2.2 [Analysis of algorithms and problem complexity]: Nonnumerical Algorithms and Problems-Sequencing and scheduling

\section{General Terms}

Theory, Algorithms

\section{Keywords}

DEVS, uncertainty, metrology, time interval

\section{INTRODUCTION}

Discrete-event simulation (DES) is a technique in which the simulation engine plays a history following a chronology of events. The technique is called "discrete-event" because the processing of each event of the chronology takes place at discrete points of a continuous time-line and takes no time with respect to the virtual simulated time (even though it may take a non-negligible time to compute with respect to the real wall-clock time).

One of many usages of DES is being part of a decisionmaking process for industrial and research works. Here, data is collected from the real system using metrological instruments and processes. From them, a set of measurement results is obtained and used as input to feed the simulation.

Modern metrology processes [3] state that it is not possible to determine a unique true value for a measurand and all measurement results require a quantification of its uncertainty to be complete.

The Bureau International des Poids et Mesures (BIPM) defines in [3] the proper procedures to obtain measurement results and the basic concepts behind them. The measurement result with uncertainty quantification is usually represented as an uncertainty interval with a certain level of confidence associated. We will not enter in the details of how the mea- 
surement result is obtained in this paper, but rather in how to use it for simulation.

In the context of DES, we consider the result of a simulation to be a trajectory of states or outputs describing the dynamic behavior of the model. In DES a small change in the timing of an event may produce a different state or output. Having uncertainty about the time of the input events may produce a tree-like result (with uncertainty time component) in place of a linear sequence of states.

As far as we know, no work has been done in DES to obtain all possible trajectories using a set of measured events as inputs and considering their timing uncertainty quantification.

We have special interest in the set of DES models described by the Discrete Event System Specification (DEVS) formalisms family and reusing previously defined models. We set our goal to provide the means to obtain all the possible state and output trajectories (as a tree) for DEVS models.

Our scientific contribution here are the abstract algorithms to simulate DEVS models with input events having uncertainty quantification of the time component. We provide the characterization of a subclass of DEVS models that can be simulated forking finite processes in each simulation step by the previously mentioned algorithms, the Finite Forkable DEVS (FF-DEVS).

We describe the limitations of the FF-DEVS subclass and show examples of DEVS models that are part or not of this subclass. We compare FF-DEVS against other subclasses defined in the literature.

We end our presentation of FF-DEVS with a case study showing the application of the algorithms simulating a trafficlight with a pedestrian call button. We present the trajectories obtained based on different input values and accuracy levels and discuss the potential of this method.

\section{BACKGROUND}

\subsection{Discrete-Event System Specification (DEVS)}

Among the many Discrete-Event Simulation techniques, our focus is on the DEVS formalism [19, 18], which provides a theoretical framework to think about Modeling using a hierarchical, modular approach. The modeling hierarchy has two kinds of components: atomic models and coupled models.

In DEVS two kinds of events are supported:

- Exogenous events: occurrence of an input at a given simulation time.

- Endogenous events: occurrence of an internal transition after having no exogenous events for a given simulation time.

The abstract simulator for DEVS defined in [ZPK+76, ZPK00] is divided in three components named simulator (for atomic models), coordinator (for coupled models) and root-coordinator for the main loop of the simulation.
Multiple extensions had being defined for this formalism in the past few decades. We find particularly relevant for our work the subclasses Schedule Preserved DEVS (SP-DEVS) [9] and Finite \& Deterministic DEVS (FD-DEVS) [10].

Both extensions, SP-DEVS and FD-DEVS, subclass DEVS for researching state reachability. In some sense, our problem can be thought as finding state reachability bounded to time intervals and a selection of messages.

The FD-DEVS subclass is restricted to those models having a finite set of states and scheduling transitions expressed by rational time advances.

The SP-DEVS subclass, which subclasses FD-DEVS, is restricted to the models that do not change scheduled transition times when receiving an exogenous event.

\subsection{Metrology}

For the metrology concepts and terminology used in this paper, we follow Guide to expression of Uncertainty in Measurement [3] and International Vocabulary of Metrology [4] documents provided by Bureau International de Poids et Mesures (BIPM).

Mostly, we only make reference to three metrological terms:

- Measurand [4]: "quantity intended to be measured".

- Measurement Result [4]: "set of quantity values being attributed to a measurand together with any other available relevant information", "Note 2: A measurement result is generally expressed as a single measured quantity value and a measurement uncertainty".

- Measurement Uncertainty [4, 3]: "non-negative parameter characterizing the dispersion of the quantity values being attributed to a measurand, based on the information used".

\subsection{Interval Arithmetic}

The measurement results can be described as intervals of real numbers. A way to properly work with them is using interval arithmetic, which describes how to operate with this kind of data [16].

In interval arithmetic, the addition of two intervals is defined as the interval where lower endpoint is defined by the addition of the two lower endpoints and higher endpoint is defined by the addition of the two higher endpoints. Similar definitions exist for other operations as subtraction, multiplication and division.

The definition of comparison operations for intervals is more complicated because the two intervals being compared may intersect, which results in multiple comparison cases that require proper handling $[1,16]$.

To reduce the amount of notation being introduced, we express the few comparisons between intervals used for the presented algorithms using comparison on their endpoint values. 


\section{RELATED WORK}

The closer match to our work are studies on state reachability tools and properties.

In [8], state reachability is studied for a subset of DEVS models having a finite set of states. An algorithm is provided to decide if a state is reachable or not when simulating a model.

In [10], Finite \& Deterministic DEVS is formally defined and algorithms to study of all reachable state based in graph theory are provided.

In [9], Scheduling Preserved DEVS is introduced as a subclass of FD-DEVS. A timed language is also introduced that allows characterization of reachable states in a timed fashion for first time.

In [18], Symbolic DEVS is proposed to study multiple trajectories of same model simultaneously using Polynomials as Time-Advance functions.

All these solutions focus on the validation of the model properties and not on the reachability based on measured data; none of them consider the introduction of uncertainty on the time of occurence of events, making this the principal distinction from our work.

Other works related to uncertainty in DES are those in the area of Logical Processes Simulation proposing the use of Approximated Time. In these works $[14,6,7,15,2]$, the main goal is to speed up simulation exploiting uncertainty. The approach is taking models defined with perfect precision and introduce small uncertainty to the time points in the time-line. Then the simulation is executed introducing uncertainty intervals such that the resulting scheduling of events can improve the parallelism of the simulation. In other words, the idea is to choose an approximation of the initial simulation that fits in the uncertainty boundaries and results in a better parallel execution. These works are far from our goal. On the contrary, we focus on obtaining all possible trajectories that may result from input events having their time of occurence defined by uncertainty intervals, but still avoiding the introduction of additional uncertainty.

As part of our solution we propose forking the simulation at some points of the time-line. This approach is not new in simulation. Some examples of its use include [17] where forking (or branching) is proposed for studying simultaneous events in DES. Other authors $[12,13,11]$ propose the use of forking (or cloning) in the context of interactive simulations where user may be undecided at some point and simulation needs to keep going. In this paper we don't go into the details of how the simulation is forked. We consider the architectures proposed in the afore-mentioned papers are good starting points for an implementation of our algorithms. However some adaptation is necessary given neither of them is based on DEVS.

\section{ALGORITHMS}

In this section we present a set of algorithms based on the classical schema proposed in [18] (root-coordinator, coordinator, simulator) allowing the use of measurement results as input for the top-level model.

The proposed algorithms compute all possible states and output trajectories considering every possible time of occurence within an uncertainty interval for each event resulting or dependent from a measurement.

\subsection{Interfacing with DEVS}

The measurement results being used as input are introduced to the simulation as an ordered queue of events where the time component is represented by a closed real interval between two finite endpoints.

Our algorithms assume that the ordering of the queue of events used as input is done first by the min-value endpoint of the time interval defined in the event and second by the max-value endpoint of the same interval.

The message component of the Events used for input is an element from the model defined input set.

While the proposed algorithms require operating using interval arithmetic for the handling of time, we do not require any modification of the DEVS models definition for operating.

Therefore, we can reuse previously defined DEVS models in new contexts were information about the uncertainty is valuable for the decision-making process and it was previously not taken into account.

\subsection{Simulator Abstract Algorithms}

In Algorithm 1 we present the abstract algorithms for simulating atomic models. The structure is similar to the DEVS abstract simulator in [18].

The first change we introduce is the use of real intervals for the $t_{\text {next }}$ and $t_{\text {last }}$ variables. At the beginning of the simulation both are discrete points in the time-line, but after an external transition is processed it may be necessary the use of proper intervals. For instance, when an external event is received in the interval $[0,1] \mathrm{sec}$., after processing the event the $t_{\text {last }}$ variable will be assigned the $[0,1]$ interval value representing the time of this last processed event.

Our INIT procedure is almost the same as the one proposed by Zeigler. Here, the difference is only the use of interval arithmetic for initializing the variables.

Our INTERNAL function, representing the *-message defined in the DEVS abstract simulator, has a few more changes than INIT. The function does not take any parameter and returns the output produced by the DEVS $\lambda(s)$ function. The validation of the parameter $t$ is delegated to the parent coordinator, so $t$ is always assumed to be valid. Compared to standard DEVS algorithm in which the only case considered is when the current time matches $t_{n e x t}$, in this interval version of the algorithm, the following simple adjustment is required: the resulting time advance is defined by an with both endpoints equal.

The INPUT function takes the role of the $x$-message defined on DEVS abstract simulator. Here, we need to branch 
the simulation execution for obtaining the trajectories consequence of each reachable state based on the uncertainty interval received as part of an input-event.

For studying the multiple branches of the simulation, we use a forking approach in which each branch is executed in a child process. Indeed, when the event is received, because of its uncertainty interval, the time elapsed in the current state when the event is processed can be any value that matches the uncertainty interval. Since $\delta_{\text {ext }}$ is a function of this elapsed time, each of these time elapsed values can lead to a different state of the simulation. Each a new branch is created to simulate each alternate time-line, continuing in one of these possible states. We break the uncertainty interval $I=\left[i_{1}, i_{2}\right]$ into segments as follows: the image of the function $f(a)=\delta_{\text {ext }}(s, a, x)$ is the set $S_{(s, x)}^{\left[i_{1}, i_{2}\right]}=\left\{S_{1}, \ldots, S_{n}\right\}$ of all possible states that can be reached from this uncertainty interval when the initial state and values are fixed to $(s, x)$. If we assume that $S_{(s, x)}^{\left[i_{1}, i_{2}\right]}$ is finite, then the interval $I$ can be split into $n=\left|S_{(s, x)}^{\left[i_{1}, i_{2}\right]}\right|$ disjoint intervals $I_{1} \ldots I_{n}$ such that $\bigcup_{i=1}^{n} I_{i}=I$ and $\bigcap_{i=1}^{n} I_{i}=\emptyset$. We call this set of sub-intervals the pre-image pieces of $f(a)$.

After all children have been forked, the parent process can be destroyed since the children cover all trajectories already.

Notice in the algorithm, we use the definition of the elapsed time as the middle position between the endpoints of the uncertainty sub-intervals, but by construction, any position in the interval would produce an identical result; this value is only chosen to simplify the case of zero-length pseudointervals (intervals reduced to a single discrete point).

In the next section we present Finite Forkable DEVS (FFDEVS), a subclass of DEVS ensuring the pimg variable used in INPUT gets always a finite set of intervals. Having finite intervals in pimg guarantees forking finite set of processes in each simulation step.

\subsection{Coordinator Abstract Algorithms}

Coordinator algorithms are slightly different than those proposed in [18]. Their definition adds little to current discussion while it would require to cover lots of technical details. Given the length restrictions for the paper, we decided to skip the coordinator algorithm definition here.

To avoid referring to these skipped algorithms, we will simulate the examples for next section using the closure resultant model [5], which does not require any coordinator for simulating the model.

\subsection{Root-Coordinator Abstract Algorithms}

The root-coordinator is in charge of running the main loop of the simulation and, in our case, introducing the measured events into the top model. For measured events, we keep a queue of input which is sorted in increasing order of the low endpoint value of each interval first, and on the high endpoint value second.

In Algorithm 2, we can see the variables defined for the root-coordinator and the main loop. Before advancing the simulation, each iteration of the main-loop has to solve col-

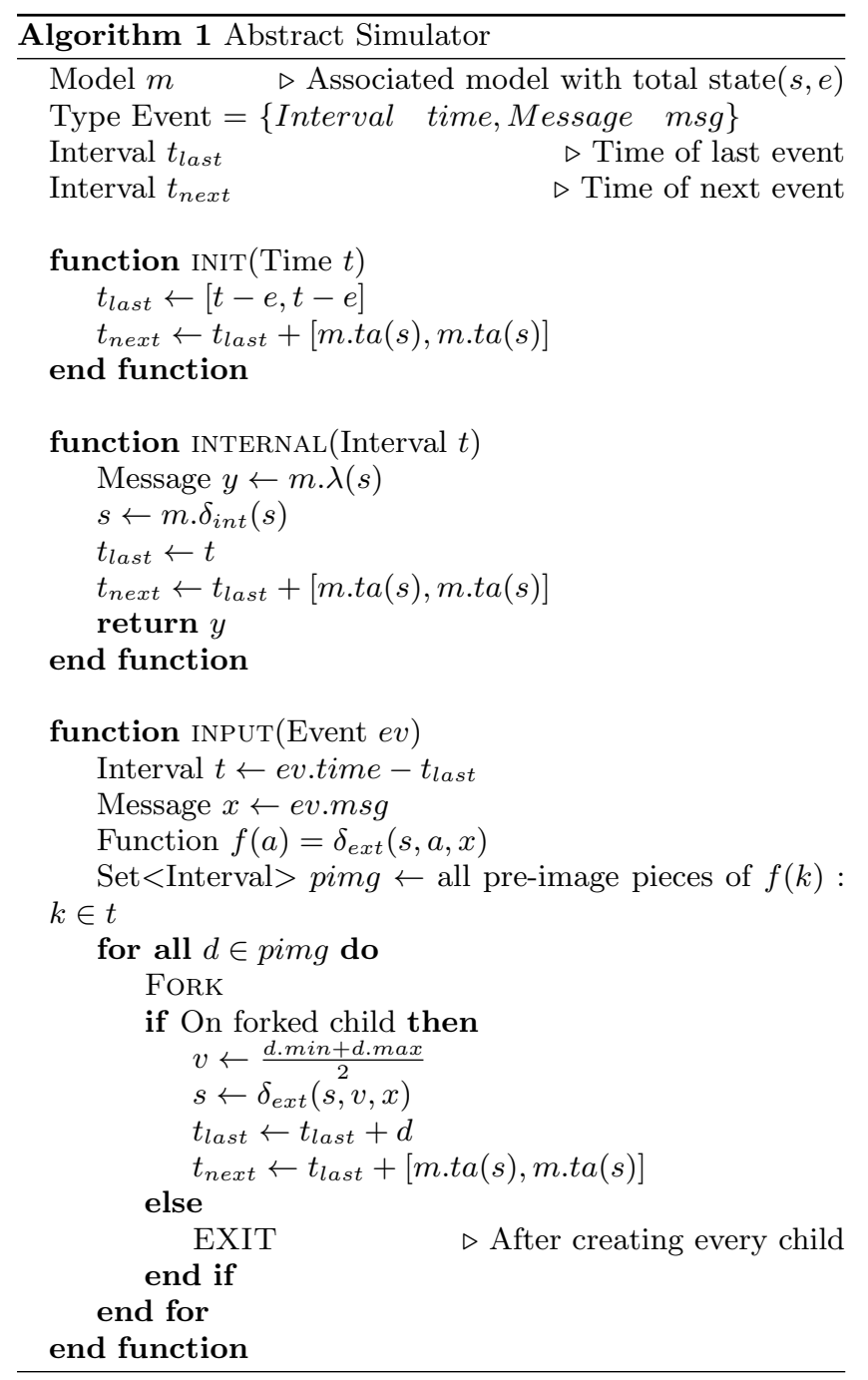

lisions between the measure event interval and the interval of the next scheduled transition. We advance the simulation differently depending on which one of three following scenarios applies:

- No collision is found: a scheduled transition or an input event is ready to be processed and its time interval does not intersect any other event.

- Input collision: a collision is found involving an input event and either another input event or the scheduled transition in the top model, and the colliding input event is scheduled earlier than the earliest endpoint of the scheduled transition interval.

- Scheduled collision: the scheduled transition collides with a set of input-events but none of the input events is scheduled earlier than the earliest endpoint of scheduled transition interval.

Algorithm 3 shows how to advance the simulation when the next input and the next scheduled transition are not overlapping with each nor with other events in the input queue.

In Algorithm 4 we consider the second scenario where a collision happens before the start of the interval for the next 


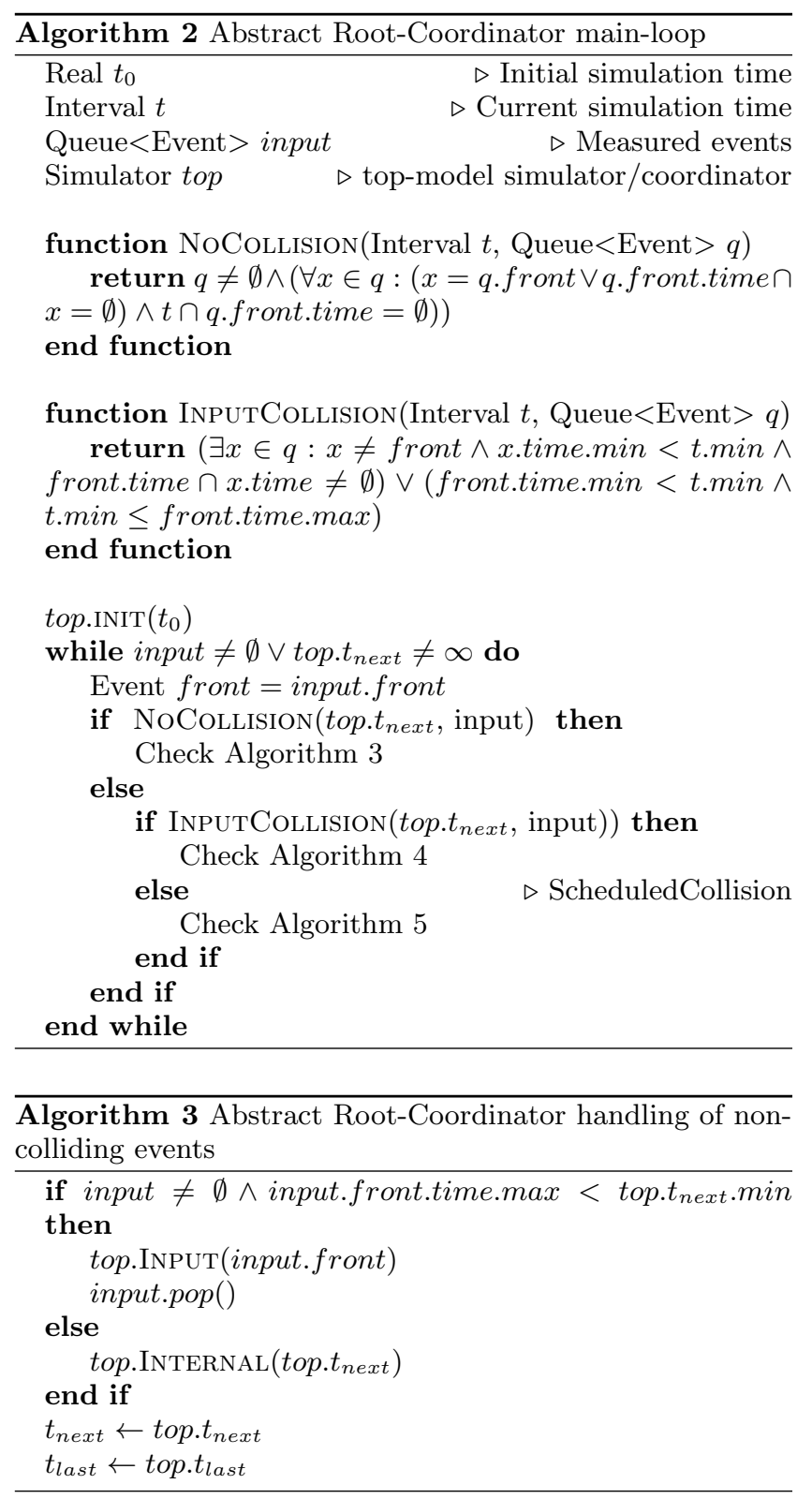

scheduled transition.

We break down this case into two complementary sub-cases: either one at least of the colliding input-events final endpoint comes earlier than the starting endpoint of the scheduled transition interval, or all the colliding input-events final endpoints come after the starting endpoint of the scheduled transition interval.

For the first sub-case, we create a set with all colliding inputevents for which the interval starting endpoint comes before the final endpoint of the interval of the event interval ending the first and define this end as limit for execution. For each event in the set we fork a new process to continue the simulation, we simulate the input of each input-event between the times each event interval starts and the limit and we remove the event from the input-queue. The parent process is ended after creating all the forks since they cover all the possible simulation branches.

For the second sub-case we follow a similar idea, but this time the limit is set to the starting endpoint of the scheduled transition interval. Also, we need to consider the possibility that none of the events is introduced before the scheduled transition is executed. For this reason, we keep the parent process alive and we rewrite in the queue every input that was included in the set to start their intervals at the same time as the limit.

When the input is processed, rescheduling of the internal transition happens making it impossible to cut in pieces all inputs at once.

The forking here is finite, because the number of input is finite and there is only one scheduled transition, which lead to forking at most a number of processes equal to the number of measured input-events plus one.

It is important in both sub-cases to rewrite the remaining events on the input queue after processing any event, to preserve the causality chains.

Finally, in Algorithm 5 we consider the last scenario in which a collision happens inside the scheduled transition interval.

This case is also broken down into two smaller sub-cases: when at least one input interval ends before the end of the scheduled transition interval and when all input event intervals end after the end of the scheduled transition interval.

The main difference with the previous scenarios is the possibility of running the scheduled transition before each input. In this case, an extra child is forked to process the scheduled transition. In the new child, legit DEVS models guarantee that after finite scheduled transitions, the model will change the time for the scheduled transition making the input be processed by previous scenario.

Meanwhile, those children produced by executing the input modify the scheduled transition making it obsolete giving no need to run it anymore.

\section{FINITE FORKABLE DEVS}

In this section, we present Finite Forkable DEVS (FF-DEVS), a subclass of DEVS models. This subclass of models can be simulated using the previously defined algorithms for which it guarantees that each simulation step will require a finite number of fork operations.

It is important to notice that having a finite number of fork operations is not the same as having a finite trajectories. Our models are allowed to generate infinite trajectories, and they do it for any input event being introduced with its time component described by a proper interval.

The abstract simulator algorithms proposed in previous section only require forking the simulation when an external transition is executed. The input event time component being an uncertainty interval provides infinite continuous values that need to be evaluated by the external transition to obtain all possible states. 


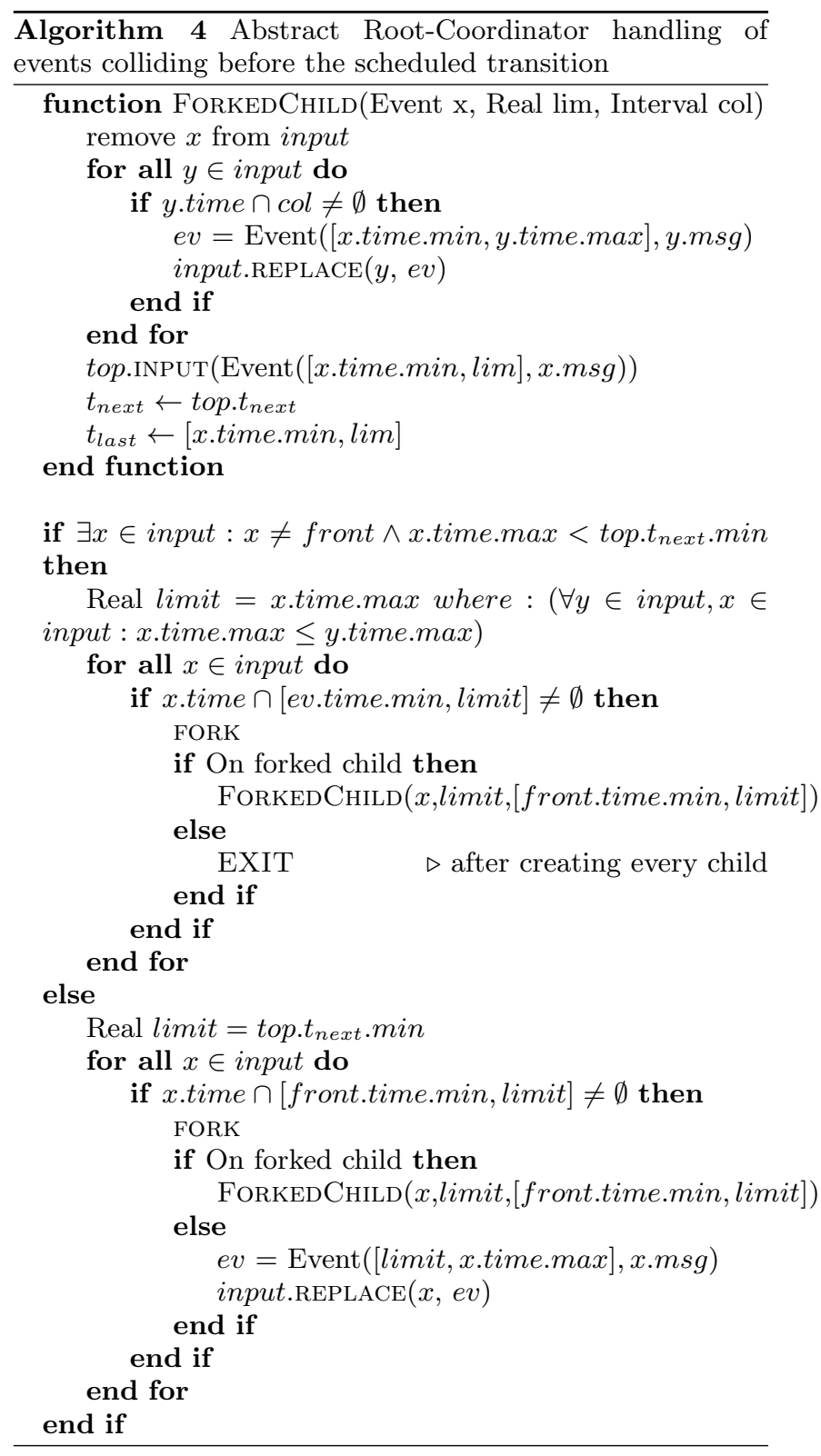

Our proposed solution is to break a given continuous interval into smaller intervals for which the image of the external transition function is a unique state value. Also, we want the number of resulting intervals to be finite, as previously stated. This requires to set a restriction on the set of valid external transition functions to those being described by a constant piece-wise function of the elapsed time variable after the state and input parameters are fixed.

Symbolically, we define an FF-DEVS Atomic model as the structure $\mathrm{A}=<\mathrm{X}, \mathrm{S}, \mathrm{Y}, \delta_{i n t}, \delta_{e x t}, \lambda, \mathrm{TA}>$ where:

$\mathrm{X}$ is the set of input values

$\mathrm{S}$ is the set of states

$\mathrm{Y}$ is the set of output values

$\delta_{\text {int }}: S \Rightarrow S$ is the internal transition function

$\delta_{\text {ext }}: Q \times X \Rightarrow S$ is the external transition function where:

$Q=(s, e): s \in S, 0 \leq e \leq T A(s)$ is the total set

$e$ is the time elapsed since last transition

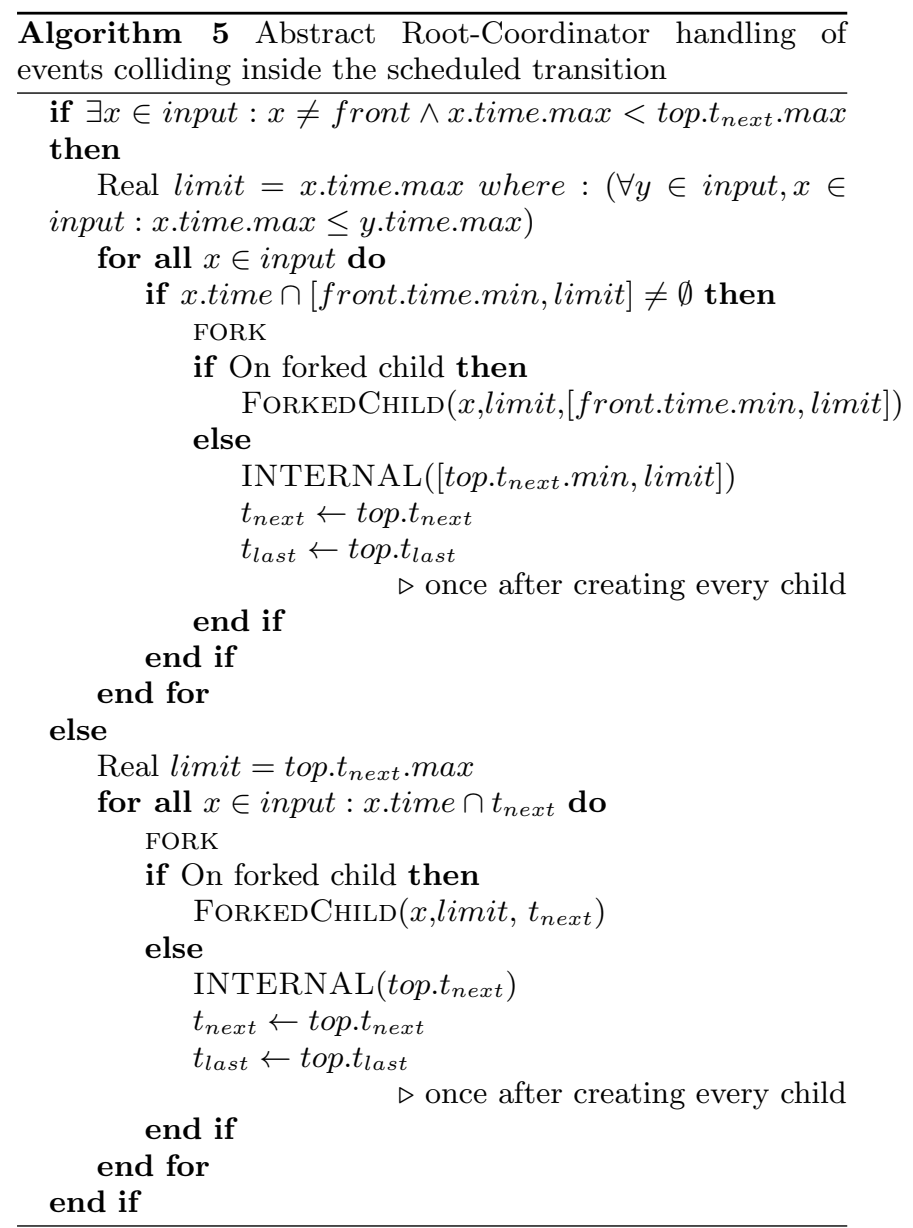

and $\forall s^{\prime} \in S, \forall x^{\prime} \in X, \forall k<\infty+: e<k \Rightarrow \delta_{\text {ext }}\left(s^{\prime}, e, x^{\prime}\right)$ is a constant piece-wise function described by finite pieces $\lambda: S \Rightarrow Y$ is the output function $T A: S \Rightarrow R_{0, \infty}^{+}$is the time advance function

When advancing the simulation a single step, the proposed restriction is certainly sufficient to ensure only the behavior of a finite set of domain segments is needed. In future work, we should evaluate if the restriction is necessary or it may be relaxed somehow.

Notice that any model defined in FF-DEVS is necessarily a DEVS model and it requires no change at all in its definition to be used as such. The other way around does not work and we will show examples of models in co-FF-DEVS later in this section.

The coupled models used in DEVS are all valid models in FF-DEVS, as far as the atomic components used are FFDEVS models. Indeed, coupling has no impact on the way the events need to be placed in the time-line; it may introduce new overlapping of intervals, but such overlapping is already handled by the algorithm.

In the case a coupled model is routing events with uncertainty intervals that collide, the coordinator routing the events should break their uncertainty intervals into smaller pieces as showed in the root-coordinator algorithms. 
We said earlier, when presenting the algorithms, that coordinators are not required for running the simulation because of the closure property. We know DEVS models are closed under coupling, meaning that a single DEVS atomic model can represent any coupled DEVS model.

Given that FF-DEVS models are valid DEVS models, we know then that a single DEVS atomic model can represent any FF-DEVS model. To assert the FF-DEVS subclass is also closed under coupling, we need to validate that the atomic model produced by the closure does not require an external transition function that does not cover the previously stated restriction.

We can think the state of the equivalent atomic model of a FF-DEVS model as the tuple having in each position a corresponding state to the one of each atomic model in the original model and the external transition function as a two step process, first processing the routing of the input to know which state components will be modified and then calling the corresponding original external transition functions to modify each of the state components.

The new external transition function will have then constant piece-wise behaviour too. The quantity of pieces will be incremented when the pieces endpoints do not match as in the following example.

Model A:

$$
\delta_{\text {ext }}(s, e, x)= \begin{cases}1 & 0 \leq e \leq 1 \\ 2 & e>1\end{cases}
$$

Model B:

$$
\delta_{\text {ext }}(s, e, x)= \begin{cases}1 & 0 \leq e \leq 2 \\ 2 & e>2\end{cases}
$$

A composed version, where both models are connected to the EIC and no internal couplings are defined would be the following:

Composed model:

$$
\delta_{\text {ext }}(s, e, x)= \begin{cases}(1,1) & 0 \leq e \leq 1 \\ (1,2) & 1<e \leq 2 \\ (2,2) & e>2\end{cases}
$$

While the original external transition functions have each a max of two intervals of pre-image, the new composed External Transition Function has the possibility of three. We can bound the quantity of intervals produced by the composition, and then since they are constant piece-wise and finite when evaluated in a finite interval, we can say the atomic equivalent covers the requirements to be a FF-DEVS model too.

To get a better idea of the practical limitations of FF-DEVS modeling, we will present a list of classical models used in the literature and discuss for each of them if they belong or not to the FF-DEVS models subclass.

- Passive[18]

This model never produces an output. Since output is never produced, there is no necessity of more than a single unique state. If the State set has a single element the external function has to be a constant function. A constant function (a constant piece-function of a single piece) covers the requirements to be in FF-DEVS.

- Storage[18]

This model is used to simulate a memory cell capable of storing a single value. The model can receive two kinds of events, one asking for the last stored value and the other asking to store a new value. In the case a request for the stored value is received, the state is changed to a new one having the value to be output and scheduling the next internal transition after zero-time. In the case the a store event is received, the value in the message is stored as the state of the model and the model becomes passive. Both possibilities are independent of the elapsed time variable, and then after state and input parameters are fixed for the external transition function we obtain a constant function, which covers the requirements for the model being in FF-DEVS.

- Counters

In [18] three counter models are described: binary counter, n-ary counter and infinite counter.

The n-ary counter only outputs a predefined message after receiving $\mathrm{n}$ messages. To do so, the state represents a counter between 0 and $\mathrm{N}$, every time a message is processed by the external transition, the counter is incremented. If the current state is below $\mathrm{N}$, the model is passive, and when it is at $\mathrm{N}$ the model next internal transition is scheduled in zero-time. The internal transition resets the counter to zero before the output was sent. In any case, the external function is independent of the elapsed variable, then it is a FF-DEVS models. The binary counter is a particular case of the n-ary counter, on which $\mathrm{n}$ is equal to two.

The infinite counter is similar to the storage model, in the sense it can receive two kinds of events, one incrementing the counter and the other asking for the current count and resetting the counter. Here, the counter state is a pair of current count and if the value was requested, when the value is requested a zero-time transition is scheduled to output and reset the counter. This model is also a FF-DEVS model for the same reason as previous ones, external transition is independent of the elapsed time variable.

- Accumulator

This is a slight variation of the infinite counter model, in place of counting the messages, it receives messages with numbers and it sums them into the current state. This more interesting model to study is also into the FF-DEVS models class.

Previous models are all instances of the FF-DEVS subclass. We present now some examples of models not in the FFDEVS subclass.

- Generator [18]

This model is used to generate outputs of predetermined values at fixed points of time. The model input set is empty and the external transition function is expected to never execute. Anyway, its external 
transition function is required to be defined properly even when no input will produce any interesting behavior. Here, the external transition reschedules the next internal transition at the same absolute time it was scheduled before, but to do so, the time advance needs to be defined as the difference between the previous time advance and the elapsed time. After fixing the state and the input in the external transition function, the function is linear in the elapsed time, which is not a suitable behavior for a valid FF-DEVS model. Allowing this kind of behavior would require infinite forks if using our algorithms.

\section{- Processor [18]}

This model receives a jobs to be performed, each process takes a fixed period of time to be executed. In the case the processor is busy and a new job is received, the new one is queued until current one is completely processed. After processing each job, an output is generated to inform the processing was completed. The external transition function for this model is also linear in the elapsed time for some combinations of input and state values. Here, when the queue is not empty, the introduction of a new job requires the use of the difference between current time advance and elapsed time as in the generator, making it a non FF-DEVS model.

- SP-DEVS Traffic Light [9]

This model represents a traffic light with a pedestrian call button that never changes a scheduled internal transition. When the button is pressed by, the event is registered into the state and when the previously scheduled internal transition is reached its value is used for deciding the next state, for example to decide next light to be turn on. This is pretty similar to what done in the previous two examples, and has the same characteristic of being linearly dependent on the elapsed time for external transitions making it an invalid FFDEVS model.

To summarize, the Passive, Storage, and Counter models are examples of models included in FF-DEVS, the Generator, Processor, and SP Traffic Light are examples of models in DEVS but not in FF-DEVS.

Comparing against other subclasses of DEVS, we can say that FF-DEVS is not strictly included in SP-DEVS [9] or FD-DEVS [10] since neither of them include models with infinite set of states as the infinite counter included in FFDEVS. Also, we can say that neither SP-DEVS nor FDDEVS are strictly included in FF-DEVS since we can find models, like the Generator, that are included in both of them but not in FF-DEVS. However, the intersection between these classes is not empty. Some models, like the binary-counter for example, belong to both FF-DEVS and FD-DEVS, while some others, like the Passive model, belong to all three of them.

\section{CASE STUDY}

In this section we study the simulation of a traffic light FFDEVS model. This model is used here to exemplify the usage of the simulator and the kind of results to be expected.
For the same model, we show the results obtained when not using the uncertainty quantification, and then using intervals of different length as those that can be obtained when increasing or decreasing the accuracy of the instruments used for data collection.

The traffic light we are modeling is required not to be schedule preserving in order to be a valid FF-DEVS model as we showed in previous section.

The following is the expected behavior of the model:

- When a pedestrian pushes the button while the traffic light is on red, it will change to white after 5 seconds.

- When a pedestrian pushes the button while the traffic light is on white, it will stay on white for 15 more seconds.

- While the button is not pushed, the traffic light will iterate 45 seconds on red, 15 seconds on white.

- The initial state of the simulation has the red light on with 0 seconds of elapsed time.

We can see in Algorithm 6 how the internal and external transitions can be implemented for this model.

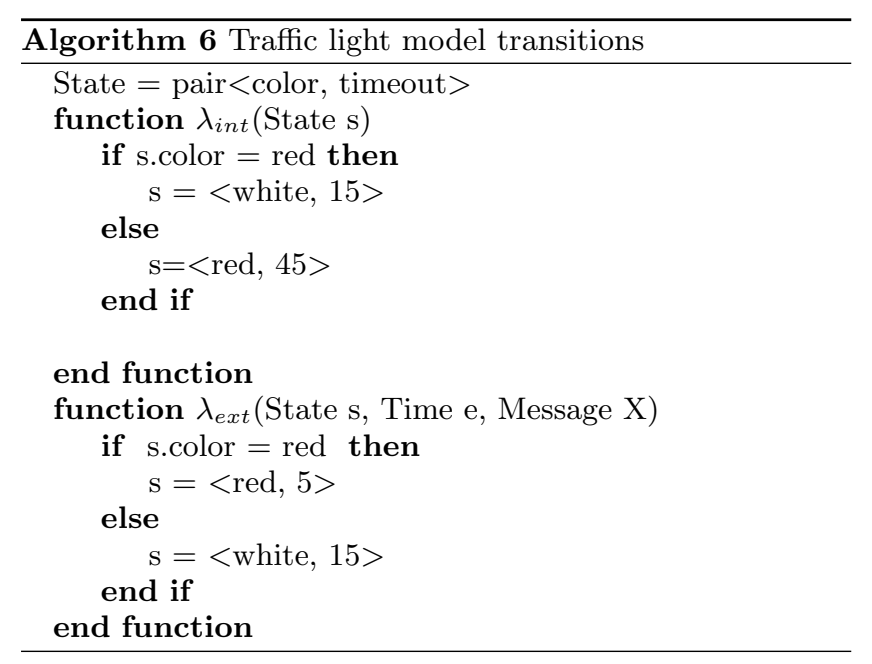

In Figure 1, we show the trajectories of a traffic light with no input at all. In this case, the simulation iterates red and white using the predefined times of 45 seconds and 15 seconds.

In Figure 2, we show the trajectories of a traffic light with pedestrians pushing the button at 5 and 71 seconds from the start of the simulation with no uncertainty intervals. Therefore, we know that at 71 seconds the traffic light will be on red and wait 45 seconds before turning back to white.

In Figure 3, we repeated the previous simulation but this time using intervals of one second length for the input. Here, most of the time we are certain about the traffic light color, but for some moments we are in an uncertain color. For instance we cannot assert the light will be red at 71.5 seconds based in the collected data. 


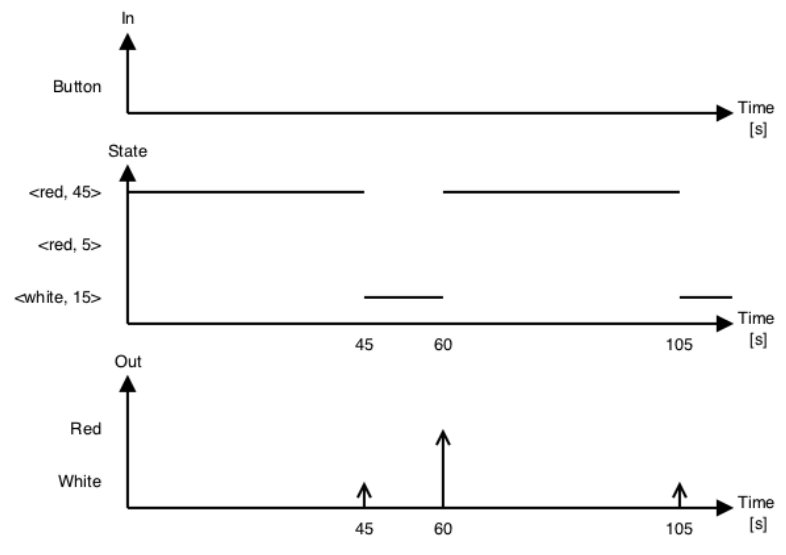

Figure 1: Trajectories of traffic light with no input

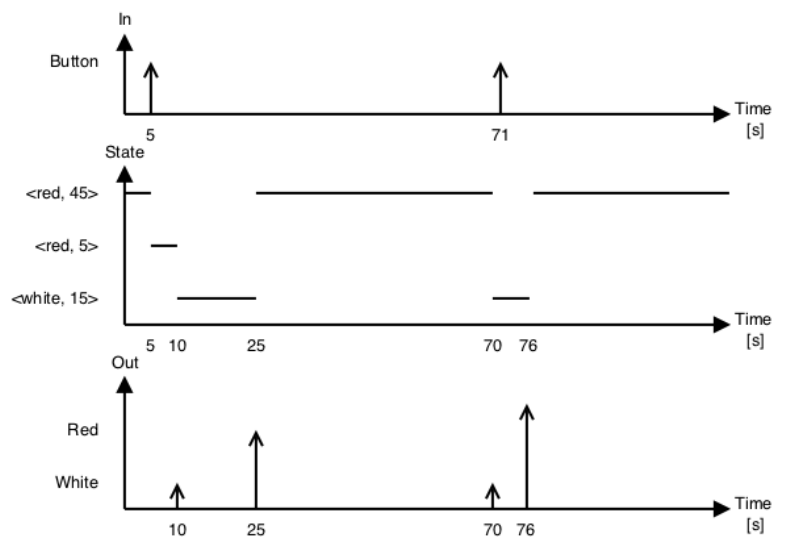

Figure 2: Trajectories of traffic light with input without uncertainty intervals

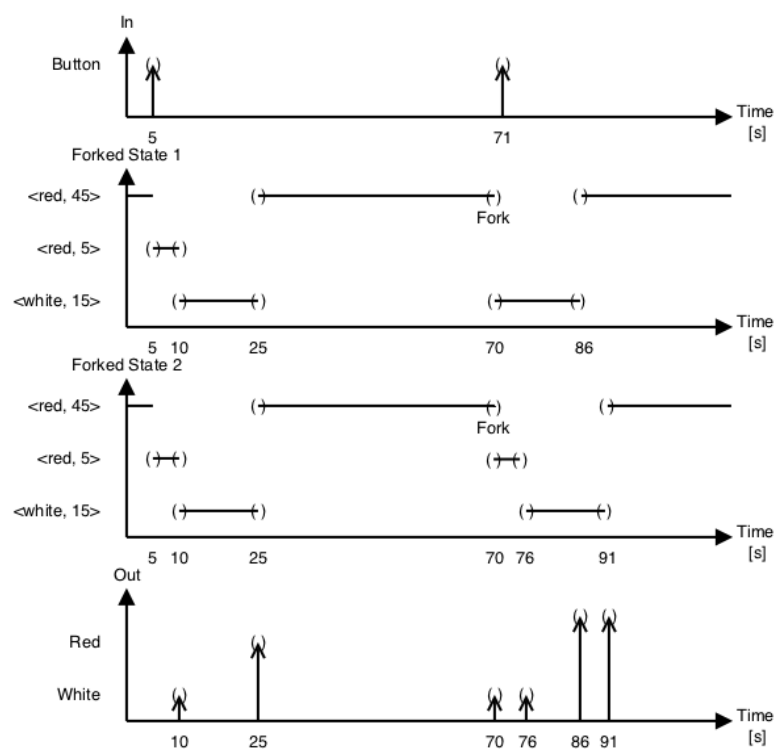

Figure 3: Trajectories of traffic light with input of 1 second accuracy

Something we would like to have in the future is a mech- anism to trace each conflict back to their originating measurement, and be able to state what accuracy is needed to remove the ambiguity from the results to a level acceptable for the decision-making process. Having this kind of information can be used to replace instruments used in repetitive processes, for example in quality control jobs.

\section{CONCLUSIONS}

We described the algorithms to simulate a subclass of DEVS models accepting input values measured with quantified uncertainty. Our algorithms produce all possible state and output trajectories for each possible value on the uncertainty intervals being used as input; this production only requires the addition of a finite set of simulators at each simulation step when simulating FF-DEVS models.

We characterized the DEVS subclass of FF-DEVS models and showed examples of which models belong to this subclass. We compared FF-DEVS subclass against other known DEVS subclasses. We provided a case study to show how to use and what to expect from the algorithms as output.

In our future work, we plan to explore new ideas to cover a larger subclass of DEVS models. We also want to work on a modeling language to simulate the introduction of uncertainty, for instance to introduce sensor models in the simulation. We want to provide the mechanisms for tracing back original measured input that resulted in a simulation branch. We want to explore optimization ideas to reduce the quantity of forks needed to simulate an FF-DEVS model using the proposed algorithms. Finally, we set our future goal to introduce uncertainty descriptions to the model. This addition will allow exploiting the algorithms in new contexts were uncertainty is a characteristic of the system and not just an input.

\section{REFERENCES}

[1] O. Aberth. Computable Calculus. Academic Press, 2001.

[2] R. Beraldi, L. Nigro, and A. Orlando. Temporal uncertainty time warp: an implementation based on java and actorfoundry. Simulation, 79(10):581-597, 2003.

[3] BIPM. Guide to the expression of uncertainty in measurement, (1995), with supplement 1, evaluation of measurement data, jcgm 101: 2008. Organization for Standardization, Geneva, Switzerland, 2008.

[4] I. BIPM, I. IFCC, I. IUPAC, and O. ISO. The international vocabulary of metrology - basic and general concepts and associated terms (vim), 3rd edn. jcgm 200: 2012. JCGM (Joint Committee for Guides in Metrology), 2008.

[5] A. Chow, B. P. Zeigler, and D. H. Kim. Abstract simulator for the parallel devs formalism. In $A I$, Simulation, and Planning in High Autonomy Systems, 1994. Distributed Interactive Simulation Environments., Proceedings of the Fifth Annual Conference on, pages 157-163. IEEE, 1994.

[6] R. M. Fujimoto. Exploiting temporal uncertainty in parallel and distributed simulations. In Proceedings of the thirteenth workshop on Parallel and distributed simulation, pages 46-53. IEEE Computer Society, 
1999.

[7] R. M. Fujimoto. Parallel simulation: parallel and distributed simulation systems. In Proceedings of the 33nd conference on Winter simulation, pages 147-157. IEEE Computer Society, 2001.

[8] A. Hernandez and N. Giambiasi. State reachability for devs models. In Proc. of Argentine Symposium on Software Engineering, 2005.

[9] M. H. Hwang and S. K. Cho. Timed behavior analysis of schedule preserved devs. In Proceedings of 2004 Summer Computer Simulation Conference, pages 26-29, 2004.

[10] M. H. Hwang and B. P. Zeigler. A modular verification framework based on finite \& deterministic devs. SIMULATION SERIES, 38(1):57, 2006.

[11] M. Hybinette and R. Fujimoto. Cloning: a novel method for interactive parallel simulation. In Proceedings of the 29th conference on Winter simulation, pages 444-451. IEEE Computer Society, 1997.

[12] M. Hybinette and R. M. Fujimoto. Cloning parallel simulations. ACM Transactions on Modeling and Computer Simulation (TOMACS), 11(4):378-407, 2001.

[13] M. Hybinette and R. M. Fujimoto. Scalability of parallel simulation cloning. In Simulation Symposium, 2002. Proceedings. 35th Annual, pages 275-282. IEEE,
2002.

[14] M. L. Loper and R. M. Fujimoto. Pre-sampling as an approach for exploiting temporal uncertainty. In Proceedings of the fourteenth workshop on Parallel and distributed simulation, pages 157-164. IEEE Computer Society, 2000.

[15] M. L. Loper and R. M. Fujimoto. A case study in exploiting temporal uncertainty in parallel simulations. In Parallel Processing, 2004. ICPP 2004. International Conference on, pages 161-168. IEEE, 2004.

[16] R. E. Moore. Interval analysis, volume 4. Prentice-Hall Englewood Cliffs, 1966.

[17] P. Peschlow and P. Martini. A discrete-event simulation tool for the analysis of simultaneous events. In Proceedings of the 2nd international conference on Performance evaluation methodologies and tools, page 14. ICST (Institute for Computer Sciences, Social-Informatics and Telecommunications Engineering), 2007.

[18] B. P. Zeigler, H. Praehofer, and T. G. Kim. Theory of modeling and simulation: integrating discrete event and continuous complex dynamic systems. Academic press, 2000.

[19] B. P. Zeigler, H. Praehofer, T. G. Kim, et al. Theory of modeling and simulation, volume 19. John Wiley

New York, 1976. 